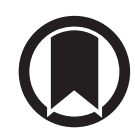

CrossMark

\section{Cancer prevalence is increased in females with sleep apnoea: data from the ESADA study}

\author{
To the Editor:
}

There is growing, but debatable evidence for the potential association between obstructive sleep apnoea (OSA) and cancer [1-10]. Available studies have reached contradictory conclusions due to limited sample sizes, and poor characterisation of OSA phenotypes or type of malignancies (all types [1-4] or site specific [6-8]). Several hypotheses have been formulated proposing why carcinogenesis can occur in the context of OSA, including older age, sleep deprivation [11,12] and concomitant obesity [13]. Intermittent hypoxia and sleep fragmentation may play a significant role via alterations in angiogenesis, sympathetic outflow, or modulation of immune function and tumour microenvironment [11, 12]. Gender-specific differences in the association between OSA and cancer prevalence have been poorly studied.

The European Sleep Apnoea Database (ESADA) is a multicentre, multinational study in which sleep laboratories recruit patients with suspected OSA [14]. The purpose of this analysis was to explore the cross-sectional association between the burden of OSA, intermittent hypoxia and cancer prevalence in the ESADA population after controlling for available recognised risk factors for cancer development. Patients older than 18 years enrolled in ESADA between 2007 and 2016 were considered. OSA was assessed by means of a sleep study (either polysomnography (PSG) or polygraphy (PG)) in accordance with the clinical routine of each participating centre $[14,15]$.

In the statistical analysis, quantitative variables are presented as mean \pm SD. Anthropometric and sleep variables in patients with or without cancer were compared using either a t-test or the Mann-Whitney U-test. The Chi-squared or Fisher's exact test were used to compare discrete variables. OSA severity was characterised by the apnoea-hypopnoea index (AHI), oxygen desaturation index (ODI), mean and lowest oxygen saturation $\left(\mathrm{SpO}_{2}\right)$ recorded via pulse oximetry during the study, and time with $\mathrm{SpO}_{2}<90 \%(\mathrm{CT} 90 \%)$ as continuous variables. AHI was stratified as $<5$ (no OSA); 5 to $<15$ (mild OSA); 15 to $<30$ (moderate OSA) and $\geqslant 30$ per h (severe OSA). Other OSA severity indices were categorised according to quartiles. Multivariate logistic regression analysis was used to evaluate association between cancer diagnosis and different OSA severity measures, expressed as odds ratios and $95 \%$ confidence intervals with continuous variables presented per 10 -unit increase. The analysis was adjusted for potential confounders and cancer risk factors: age, gender, body mass index (BMI), smoking and alcohol use. A separate analysis was performed across groups stratified for gender. A p-value of $<0.05$ was considered statistically significant.

Of 19556 patients, 388 (2\%) had been diagnosed with malignancy (prevalent cancer) (1.7\% males and $2.8 \%$ females). Patients with malignancy and AHI $\geqslant 5$ per $\mathrm{h}(\mathrm{n}=318)$ were older $(60.8 \pm 10.4$ versus $53.5 \pm 12.1$ years; $\mathrm{p}<0.001$ ) and were slightly less centrally obese (waist-to-hip ratio $0.96 \pm 0.08$ versus $0.98 \pm 0.08 ; \mathrm{p}<0.001)$ than OSA patients without malignancy. Current and previous smoking history was reported in $14.8 \%$ and $45.0 \%$ of OSA patients with malignancy, respectively. Cardiovascular and metabolic comorbidity did not differ between OSA patients with cancer versus those without cancer, despite a higher prevalence of stroke in cancer patients $(5.0 \%$ versus $2.4 \%$; $\mathrm{p}=0.003)$.

A cancer diagnosis was significantly associated with elevated AHI (AHI $\geqslant 5$ versus $\mathrm{AHI}<5$ per h: OR 1.35 , 95\% CI 1.02-1.79; $\mathrm{p}=0.03$ ), CT90\% as proportion of recording time (OR 1.08, 95\% CI 1-1.17; $\mathrm{p}=0.03$ ) and CT90\% in minutes (OR 1.02, 95\% CI 1-1.04; $\mathrm{p}=0.01$ ) but not ODI (OR 0.98, 95\% CI 0.9-1.02; $\mathrm{p}=0.98$ ) in

@ERSpublications

In a cross-sectional analysis of the European Sleep Apnoea Database (ESADA), cancer prevalence was higher in females with OSA and nocturnal hypoxia, but not in males. Further studies are needed to assess causality of gender, OSA and cancer incidence. http://bit.ly/2YuxyZA

Cite this article as: Pataka A, Bonsignore MR, Ryan S, et al. Cancer prevalence is increased in females with sleep apnoea: data from the ESADA study. Eur Respir J 2019; 53: 1900091 [https://doi.org/10.1183/ 13993003.00091-2019]. 
unadjusted models. Only CT90\% remained a predictor for cancer diagnosis after adjustment for age, gender, BMI, smoking and alcohol consumption (CT90\% as proportion of recording time: OR 1.1, 95\% CI $1-1.2$; $\mathrm{p}=0.04$; CT90\% in minutes: OR $1.02,95 \%$ CI $1-1.04 ; \mathrm{p}=0.02)$. In the analysis stratified by gender, increased odds ratios for cancer in different categories of OSA severity and degree of nocturnal hypoxia were identified in females but not in males (table 1). In males, ODI was close to significance, confirming previous studies indicating a stronger relationship between a cancer diagnosis and measures of intermittent hypoxia (ODI and CT90\%) rather than AHI $[1,3,10]$. When patients were stratified according to sleep study methodology, the association between OSA and cancer prevalence remained significant in females assessed by PG (AHI $\geqslant 15$ versus $<15$ per $\mathrm{h}$ : OR 2.23 , 95\% CI $1.2-0.4 .14 ; \mathrm{p}=0.01$; severe OSA versus no OSA: OR 2.97, 95\% CI 1.19-7.43; $\mathrm{p}=0.02$; 75th to 25th percentile of AHI: OR 3.63, 95\% CI 1.42-9.21; $\mathrm{p}=0.007$; ODI: OR $1.17,95 \%$ CI $1.02-1.33$; $\mathrm{p}=0.02$; 75 th to 25 th percentile of ODI: OR 3.53 , 95\% CI $1.46-$ 8.51; $\mathrm{p}=0.005)$. In female patients assessed by PSG, nocturnal intermittent hypoxia was associated with cancer prevalence (75th to 25th percentile of CT90\% in minutes: OR 3.01, 95\% CI 1.09-8.32; $\mathrm{p}=0.03$; CT90\% as proportion of recording time: OR 1.17, 95\% CI 1.01-1.36; $\mathrm{p}=0.04$ ). The different results between PG and PSG might be attributed to the fact that AHI is highly linked to intermittent hypoxia in PG, whereas AHI is partly linked to arousal in PSG [16]. Sleep quality measures like "sleep efficiency" and "total sleep time", excessive daytime sleepiness measured by Epworth Sleepiness Scale, as well as comorbidities potentially causing hypoxia, such as heart failure, chronic obstructive pulmonary disease or respiratory failure, did not modify the adjusted models after stratifying for gender and sleep study. The most prevalent cancer in women was breast cancer $(n=70,43.8 \%)$, followed by gynaecological (12.3\%) and thyroid (6.9\%) cancer, lymphoma (5.4\%), lung (4.6\%) and colon (3.1\%) cancer, and melanoma (3.1\%).

TABLE 1 Odds ratios for cancer prevalence in different obstructive sleep apnoea (OSA) categories assessed by apnoeahypopnoea index (AHI) and measures of intermittent hypoxia: differences between genders

\begin{tabular}{|c|c|c|c|c|}
\hline \multirow[t]{2}{*}{ OSA categories } & \multicolumn{2}{|c|}{ Male ( $n=13539, c=228$ ) } & \multicolumn{2}{|c|}{ Female $(n=5629, c=160)$} \\
\hline & Adjusted $\mathrm{OR}^{\#}(95 \% \mathrm{CI})$ & p-value & Adjusted $\mathrm{OR}^{\#}(95 \% \mathrm{CI})$ & $p$-value \\
\hline AHI (continuous) & $1.00(0.9-1.06)$ & 0.82 & $1.09(1.01-1.17)$ & 0.03 \\
\hline $\mathrm{AHI} \geqslant 15$ versus $<15$ & $0.85(0.6-1.18)$ & 0.34 & $1.58(1.06-2.33)^{* *}$ & 0.02 \\
\hline $\mathrm{AHI} \geqslant 30$ versus $<30$ & $0.88(0.63-1.23)$ & 0.46 & $1.51(0.99-2.29)^{* *}$ & 0.054 \\
\hline Mild (AHI 5-14.9) versus 0-4.9 & $1.07(0.63-1.81)$ & 0.8 & $1.5(0.85-2.63)$ & 0.16 \\
\hline AHI (quartiles) ${ }^{\pi 1} 7-19.4$ versus $0-6.9$ & $0.95(0.59-1.52)$ & 0.8 & $1.04(0.6-1.74)$ & 0.88 \\
\hline AHI 19.5-41.3 versus $0-6.9$ & $0.85(0.53-1.36)$ & 0.5 & $1.68(1.005-2.8)$ & 0.05 \\
\hline $\mathrm{AHI}>41.4$ versus $0-6.9$ & $0.99(0.6-1.6)$ & 0.96 & $1.5(0.83-2.75)^{* *}$ & 0.17 \\
\hline ODI (continuous) & $0.92(0.82-1.00)$ & 0.06 & $1.09(2.45-5.41)^{* *}$ & 0.01 \\
\hline$O D I \geqslant 5$ versus $<5$ & $1.46(0.99-2.15)$ & 0.05 & $0.7(0.44-1.08)$ & 0.11 \\
\hline $\mathrm{ODI} \geqslant 10$ versus $<10$ & $0.63(0.44-0.88)$ & 0.008 & $1.3(0.86-1.95)$ & 0.21 \\
\hline СT90\% (minutes) (quartiles) $)^{\pi} 0.5-7.5$ versus $0-0.4$ & $1.01(0.48-2.1)$ & 0.98 & $0.63(0.26-1.55)$ & 0.3 \\
\hline СТ90\% (minutes) $7.6-48.1$ versus $0-0.4$ & $1.09(0.52-2.29)$ & 0.82 & $2.035(0.97-4.26)^{*}$ & 0.06 \\
\hline СТ90\% (minutes) $>48.2$ versus $0-0.4$ & $1.02(0.46-2.28)$ & 0.95 & $2.16(0.95-4.92)^{*}$ & 0.07 \\
\hline СТ90\% (\% of recording time) & $1.04(0.9-1.21)$ & 0.55 & $1.17(1.04-1.32)^{*}$ & 0.01 \\
\hline СТ90\% (\% of recording time) (quartiles) ${ }^{\pi} 0.125-1.69$ versus $0-0.124$ & $1.04(0.49-2.2)$ & 0.92 & $0.59(0.23-1.51)$ & 0.3 \\
\hline СТ90\% (\% of recording time) $1.7-10.98$ versus $0-0.124$ & $1.21(0.58-2.52)$ & 0.62 & $2.25(1.08-4.67)$ & 0.03 \\
\hline СТ90\% (\% of recording time) $10.99-100$ versus $0-0.124$ & $1.32(0.61-2.86)$ & 0.5 & $2.27(1.01-5.12)$ & 0.05 \\
\hline
\end{tabular}

For continuous variables, odds ratios are presented per 10-unit increase. c: cancer patients; ODI: oxygen desaturation index; CT90\%: time with

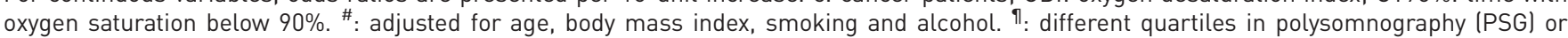
polygraphy (PG) are as follows. AHI quartiles PSG: first quartile: 0-11.4 per h; second: 11.41-25.6; third: 25.61-49; fourth: >49.1. ODI quartiles PSG: first quartile: $0-5.4$ per $h$; second: $5.41-17.5$; third: $17.51-42.1$; fourth: $>42.11$. CT90\% (in minutes) quartiles PSG: first quartile: $0-0.6$ min; second: 0.61-8.2; third: 8.21-53.3; fourth: $>53.31$. CT90\% (proportion of recording time) quartiles PSG: first quartile: 0-0.137\%; second: 0.138-1.86\%; third: $1.87-12.18 \%$; fourth: $12.19-100 \%$. AHI quartiles PG: first quartile: $0-4.3$ per $h$; second: $4.31-13.1$; third: $13.2-32.1$; fourth: >32.12. ODI quartiles PG: first quartile: 0-3.7 per $h$; second: 3.71-11; third: 11.1-29; fourth: >29.1. CT90\% (in minutes) quartiles PG: first quartile: $0-0.5 \mathrm{~min}$; second: $0.51-6.21$; third: $6.22-41$; fourth: $>41.1$. CT90\% (proportion of recording time) quartiles PG: first quartile: 0-0.1\%; second: $0.11-1.47 \%$; third: $1.48-9.33 \%$; fourth: $9.34-100 \%{ }^{*}$ : statistically significant in PSG. ${ }^{* *}$ : statistically significant in PG (refer to the text for odds ratios). 
The most prevalent cancer in males was prostate cancer $(n=56,33.1 \%)$, followed by lymphoma $(8.3 \%)$, colon cancer (8.3\%), ear, nose, and throat cancer (8.3\%), lung cancer (5.9\%) and melanoma (5.3\%). In a sub-analysis, there was no independent influence of OSA on the prevalence of breast and prostate cancer.

The main finding of our study was that OSA was associated with a cancer diagnosis, especially in females. Nocturnal hypoxia (expressed by CT90\%) associated with cancer after adjusting for potential confounders and risk factors in both genders. Our findings were in agreement with previous studies demonstrating an association between OSA and increased cancer risk [1-3]. In one study, cancer prevalence was associated with CT90\% and, in a subgroup analysis of smoking-related cancers, with ODI but not with AHI [10]. In contrast, other studies did not find an independent association between OSA and cancer incidence [2, 10], and the reasons for these differences remain unclear. Intermittent hypoxia has been linked to increased tumour proliferation and risk of metastasis. Intermittent hypoxia in OSA may elicit both preconditioning or cell death $[5,12]$. Interestingly, data on sex differences in the association between cancer and OSA remain sparse $[3,8,9]$. The prevalence of cancer subtypes in our cohort broadly mirrors that reported in Western populations. The design of our study does not allow speculations about a causal relationship between cancer prevalence and OSA. However, the observed interaction suggests a possible OSA-related mechanism in carcinogenesis, with higher susceptibility in females. Potential factors include cancer subtype, hormonal influences on both tumour cell growth and immune responses, and duration of OSA exposure, as well as gender-specific exposure patterns to cigarette smoking not fully captured in our analysis $[5,9,12]$.

The prevalence of cancer in our dataset was low (2\%) compared to previous studies [10], suggesting under-referral of cancer patients, especially those with severe or lethal cancer. This referral bias may rather underestimate the true association between OSA and cancer. Cancer patients may have a different clinical presentation with insomnia affecting the results of sleep studies. It is important to further examine the influence of OSA on cancer types. However, the small number of cases limited our analysis to breast and prostate cancer. Furthermore, adjustment was not made for important confounders of cancer risk, such as physical activity, marital status, education, shift work and genetic propensity. Our study has two major strengths: the multicentre design and the magnitude of the clinical dataset; these increase the generalisability of our results and the substantial statistical power in the gender-specific subgroup analyses.

In conclusion, our findings suggest that cancer prevalence is higher in European females with OSA. In an ongoing follow-up study, we evaluate cancer incidence, mortality, aggressiveness and effect of OSA treatment in our population.

Athanasia Pataka ${ }^{1}$, Maria R. Bonsignore $\odot^{2}$, Silke Ryan $\odot^{3}$, Renata L. Riha ${ }^{4}$, Jean-Louis Pepin ${ }^{5}$, Sofia Schiza ${ }^{6}$, Ozen K. Basoglu ${ }^{7}$, Pawel Sliwinski ${ }^{8}$, Ondrej Ludka ${ }^{9}$, Paschalis Steiropoulos ${ }^{10}$, Ulla Anttalainen ${ }^{11}$, Walter T. McNicholas ${ }^{3}$, Jan Hedner ${ }^{12,13}$ and Ludger Grote $\oplus^{12,13}$, on behalf of the ESADA study group ${ }^{14}$

${ }^{1}$ Respiratory Failure Unit, G. Papanikolaou Hospital, Aristotle University of Thessaloniki, Thessaloniki, Greece. ${ }^{2}$ Biomedical Dept of Internal and Specialistic Medicine (DiBiMIS), Section of Pneumology, University of Palermo and CNR Institute of Biomedicine and Molecular Immunology, Palermo, Italy. ${ }^{3}$ Pulmonary and Sleep Disorders Unit, St Vincent's University Hospital and School of Medicine, University College Dublin, Dublin, Ireland. ${ }^{4}$ Dept of Sleep Medicine, Royal Infirmary Edinburgh, Edinburgh, UK. ${ }^{5}$ University Grenoble Alpes, INSERM HP2 (U1042) and Grenoble University Hospital, Grenoble, France. ${ }^{6}$ Sleep Disorders Unit, Dept of Respiratory Medicine, Medical School, University of Crete, Heraklion, Greece. ${ }^{7}$ Dept of Chest Diseases, Ege University, Faculty of Medicine, Izmir, Turkey. ${ }^{8}$ 2nd Dept of Respiratory Medicine, Institute of Tuberculosis and Lung Diseases, Warsaw, Poland. ${ }^{9}$ Dept of Cardiology, University Hospital Brno and International Clinical Research Center, St. Ann's University Hospital, Brno, Czech Republic. ${ }^{10}$ Sleep Unit, Dept of Pneumonology, Democritus University of Thrace, Alexandroupolis, Greece. ${ }^{11}$ Division of Medicine, Dept of Pulmonary Diseases, Turku University Hospital and Sleep Research Centre, Dept of Pulmonary Diseases and Clinical Allergology, University of Turku, Turku, Finland. ${ }^{12}$ Pulmonary Dept, Sahlgrenska University Hospital, Gothenburg, Sweden. ${ }^{3}$ Center for Sleep and Wake Disorders, Sahlgrenska Academy, Gothenburg University, Gothenburg, Sweden. ${ }^{14} \mathrm{~A}$ list of collaborators in the ESADA study group can be found in the acknowledgements section.

Correspondence: Athanasia Pataka, Medical School, Aristotle University of Thessaloniki, Respiratory Failure Unit, G. Papanikolaou Hospital, 57010 Exohi, Thessaloniki, Greece. E-mail: patakath@yahoo.gr

Received: Oct 172018 | Accepted after revision: March 052019

Acknowledgements: The authors thank all collaborators of the ESADA study group (current and past): P. Steiropoulos, Sleep Unit, Dept of Pneumonology, Democritus University of Thrace, Alexandroupolis, Greece; J. Verbraecken, Multidisciplinary Sleep Disorders Centre, Antwerp University Hospital and University of Antwerp, Antwerp, Belgium; E. Petiet, Multidisciplinary Sleep Disorders Centre, Antwerp University Hospital and University of Antwerp, Antwerp, Belgium; G. Trakada, Pulmonary Medicine, National and Kapodistrian University of Athens, Athens, Greece; J.M. Montserrat, Hospital Clinic i Provincial de Barcelona, Barcelona, IDIBAPS Barcelona and CIBERes, Madrid, Spain; I. Fietze, Schlafmedizinisches Zentrum, Charite - University of Medicine Berlin, Germany; T. Penzel, Schlafmedizinisches Zentrum, Charite - University of Medicine Berlin, Germany; O. Ludka, Dept of Cardiology, University Hospital Brno and International Clinical Research Center, St. Ann's University Hospital, Brno, Czech 
Republic; D. Rodenstein, Cliniques Universitaires Saint-Luc, Brussels, Belgium; J.F. Masa, Hospital San Pedro de Alcüntara, Caeceres, and CIBERes, Madrid, Spain; I. Bouloukaki, Sleep Disorders Unit, Dept of Respiratory Medicine, Medical School, University of Crete, Greece; S. Schiza, Sleep Disorders Unit, Dept of Respiratory Medicine, Medical School, University of Crete, Greece; B. Kent, Guy's and St Thomas' NHS Foundation Trust, Guy's Hospital, London, UK; W.T. McNicholas, Dept of Respiratory Medicine, St. Vincent's University Hospital, Dublin, Ireland; S. Ryan, Pulmonary and Sleep Disorders Unit, St. Vincent's University Hospital, Dublin, Ireland; R.L. Riha, Dept of Sleep Medicine, Royal Infirmary Edinburgh, UK; J.A. Kvamme, Sleep Laboratory, ENT Dept, Forde Central Hospital, Forde, Norway; R. Schulz, Sleep Disorders Centre, University of Giessen, Lung Centre, Giessen, Germany; L. Grote, Sleep Disorders Center, Pulmonary Dept, Sahlgrenska University Hospital, and Center of Sleep and Wake Disorders, Sahlgrenska Academy, Gothenburg University, Gothenburg, Sweden; J. Hedner, Sleep Disorders Center, Pulmonary Dept, Sahlgrenska University Hospital, and Center of Sleep and Wake Disorders, Sahlgrenska Academy, Gothenburg University, Gothenburg, Sweden; J-L. Pepin, University Grenoble Alpes, INSERM HP2 (U1042) and Grenoble University Hospital, Grenoble, France; P. Levy, University Grenoble Alpes, INSERM HP2 (U1042) and Grenoble University Hospital, Grenoble, France; L. Lavie, Centre for Sleep Medicine, Technion Institute of Technology, Haifa, Israel; P. Lavie, Centre for Sleep Medicine, Technion Institute of Technology, Haifa, Israel; H. Hein, Sleep Disorders Center, St. Adolf Stift, Reinbeck, Germany; O.K. Basoglu, Dept of Chest Diseases, Ege University, Izmir, Turkey; M.S. Tasbakan, Dept of Chest Diseases, Ege University, Izmir, Turkey; G. Varoneckas, Institute Psychophysiology and Rehabilitation, Palanga, Lithuania; P. Joppa, Dept of Respiratory Medicine and Tuberculosis, Faculty of Medicine, P.J. Safarik University and L. Pasteur University Hospital, Kosice, Slovakia; R. Tkacova, Dept of Respiratory Medicine and Tuberculosis, Faculty of Medicine, P.J. Safarik University and L. Pasteur University Hospital, Kosice, Slovakia; R. Staats, Dept of Respiratory Medicine, Hospital de Santa Maria, Lisbon, Portugal; F. Barbe, Servei Pneumologia Hospital Arnau de Vilanova and Hospital Santa Maria, Lleida, and CIBERes, Madrid, Spain; C. Lombardi, Istituto Auxologico Italiano, IRCCS, Dept of Cardiovascular, Neural and Metabolic Sciences, St Luke Hospital, Milan and Dept of Medicine and Surgery, University of Milano-Bicocca, Milan, Italy; G. Parati, Istituto Auxologico Italiano, IRCCS, Dept of Cardiovascular, Neural and Metabolic Sciences, St Luke Hospital, Milan and Dept of Medicine and Surgery, University of Milano-Bicocca, Milan, Italy; M. Drummond, Pulmonology Dept, Hospital São João, Medicine Faculty of Porto University, Porto, Portugal; M. van Zeller, Pulmonology Dept, Hospital São João, Medicine Faculty of Porto University, Porto, Portugal; M.R. Bonsignore, Biomedical Dept of Internal and Specialistic Medicine (DiBiMIS), Section of Pneumology, University of Palermo, and CNR Institute of Biomedicine and Molecular Immunology, Palermo, Italy; O. Marrone, CNR Institute of Biomedicine and Molecular Immunology, Palermo, Italy; P. Escourrou, Service dIxplorations Fonctionnelles Multidisciplinaires Hospital Antoine Beclere, Clamart, France; G. Roisman, Unitı de Midecine du Sommeil, Hopital Antoine-Beclere, Clamart, France; M. Pretl, Centre for Sleep and Waking Disorders, Dept of Neurology, First Faculty of Medicine, Charles University, Prague, and Inspamed, Neurology and Sleep Laboratory, Prague, Czech Republic; A. Vitols, Institute of Cardiology, University of Latvia, Riga, Latvia; Z. Dogas, Sleep Medicine Center, Dept of Neuroscience, University of Split School of Medicine, Split, Croatia; T. Galic, Sleep Medicine Center, Dept of Neuroscience, University of Split School of Medicine, Split, Croatia; A. Pataka, Respiratory Failure Unit, G. Papanikolaou Hospital, Aristotle University of Thessaloniki, Greece; U. Anttalainen, Division of Medicine, Dept of Pulmonary Diseases, Turku University Hospital and Sleep Research Centre, Dept of Pulmonary Diseases and Clinical Allergology, University of Turku, Finland; T. Saaresranta, Division of Medicine, Dept of Pulmonary Diseases, Turku University Hospital and Sleep Research Centre, Dept of Pulmonary Diseases and Clinical Allergology, University of Turku, Finland; P. Sliwinski, 2nd Dept of Respiratory Medicine, Institute of Tuberculosis and Lung Diseases, Warsaw, Poland; R. Plywaczewski, 2nd Dept of Respiratory Medicine, Institute of Tuberculosis and Lung Diseases, Warsaw, Poland; P. Bielicki, Dept of Internal Medicine, Pneumonology and Allergology, Medical University of Warsaw, Warsaw, Poland.

Conflict of interest: A. Pataka has nothing to disclose. M.R. Bonsignore has nothing to disclose. S. Ryan has nothing to disclose. R.L. Riha has nothing to disclose. J-L. Pepin reports grants and research funds from Air Liquide Foundation, Agiradom, AstraZeneca, Fisher and Paykel, Mutualia, Philips, Resmed and Vitalaire, personal fees from Agiradom, AstraZeneca, Boehringer Ingelheim, Jazz Pharmaceutical, Night Balance, Philips, Resmed and Sefam. S. Schiza has nothing to disclose. O.K. Basoglu has nothing to disclose. P. Sliwinski has nothing to disclose. O. Ludka has nothing to disclose. P. Steiropoulos has nothing to disclose. U. Anttalainen has nothing to disclose. W.T. McNicholas has nothing to disclose. J. Hedner reports grants from ResMed Foundation, Philips Respironics and the European Respiratory Society, for start-up and conduct of the study. L. Grote reports grants from Resmed Foundation and Respironics Foundation, during the conduct of the study; personal fees for lecturing from Resmed, Philips and Itamar, grants from Resmed, other (equipment) from Itamar, outside the submitted work; in addition, L. Grote has a patent for sleep apnoea treatment pending.

Support statement: The ESADA network has received support from the European Union COST action B26 and is currently a Clinical Research Collaboration funded by the European Respiratory Society. Additionally, the ESADA study group has received unrestricted seeding grants from the ResMed and the Philips Respironics Foundation for the establishment of the database in 2007 and 2011. The ESADA has an ongoing collaboration with Bayer AG. Non-financial support was provided by the European Sleep Research Society and the European Respiratory Society for communication, meetings and data presentations for the ESADA collaborators.

\section{References}

$1 \quad$ Nieto FJ, Peppard PE, Young T, et al. Sleep disordered breathing and cancer mortality: results from the Wisconsin Sleep Cohort Study. Am J Respir Crit Care Med 2012; 186: 190-194.

2 Christensen AS, Clark A, Salo P, et al. Symptoms of sleep disordered breathing and risk of cancer: a prospective cohort study. Sleep 2013; 36: 1429-1435.

3 Campos-Rodriguez F, Martinez-Garcia MA, Martinez M, et al. Association between obstructive sleep apnea and cancer incidence in a large multicenter Spanish cohort. Am J Respir Crit Med 2013; 87: 99-105.

4 Martínez-García MA, Campos-Rodriguez F, Durán-Cantolla J, et al. Obstructive sleep apnea is associated with cancer mortality in younger patients. Sleep Med 2014; 15: 742-748. 
5 Marshall NS, Wong KK, Cullen SR, et al. Sleep apnea and 20-year follow-up for all-cause mortality, stroke, and cancer incidence and mortality in the Busselton health study cohort. J Clin Sleep Med 2014; 10: 355-362.

6 Chen JC, Hwang JH. Sleep apnea increased incidence of primary central nervous system cancers: a nationwide cohort study. Sleep Med 2014; 15: 749-754.

7 Martinez-Garcia MA, Campos-Rodriguez F, Nagore E, et al. Sleep-disordered breathing is independently associated with increased aggressiveness of cutaneous melanoma: a multicenter observational study in 443 patients. Chest 2018; 154: 1348-1358.

8 Chang WP, Liu ME, Chang WC, et al. Sleep apnea and the subsequent risk of breast cancer in women: a nationwide population-based cohort study. Sleep Med 2014; 15: 1016-1020.

9 Gozal D, Ham SA, Mokhlesi B. Sleep apnea and cancer: analysis of a nationwide population sample. Sleep 2016; 39: $1493-1500$.

10 Kendzerska T, Leung RS, Hawker G, et al. Obstructive sleep apnea and the prevalence and incidence of cancer. CMAJ 2014; 186: 985-992.

11 Gozal D, Farré R, Nieto FJ. Putative links between sleep apnea and cancer: from hypotheses to evolving evidence. Chest 2015; 148: 1140-1147.

12 Almendros I, Montserrat JM, Torres $\mathrm{M}$, et al. Obesity and intermittent hypoxia increase tumour growth in a mouse model of sleep apnea. Sleep Med 2012; 13: 1254-1260.

13 Park J, Morley TS, Kim M, et al. Obesity and cancer - mechanisms underlying tumour progression and recurrence. Nat Rev Endocrinol 2014; 10: 455-465.

14 Hedner J, Grote L, Bonsignore M, et al. The European Sleep Apnoea Database (ESADA): report from 22 European sleep laboratories. Eur Respir J 2011; 38: 635-642.

15 Iber C, Ancoli-Israeli S, Chesson A, et al. The AASM Manual for the Scoring of Sleep and Associated Events: Rules, Terminology and Technical Specifications. 1st Edn. Westchester, American Academy of Sleep Medicine, 2007.

16 Escourrou P, Grote L, Penzel T, et al. The diagnostic method has a strong influence on classification of obstructive sleep apnea. J Sleep Res 2015; 24: 730-738. 